Functional Coherence of Molecular

Networks in Bioinformatics 

Mehmet Koyutürk • Shankar Subramaniam Ananth Grama

Editors

\section{Functional Coherence of Molecular Networks in Bioinformatics}

望 Springer 


\section{Editors}

Mehmet Koyutürk

Department of Electrical Engineering and Computer Science

Case Western Reserve University

10900 Euclid Ave.

Cleveland Ohio 44106

USA

mxk331@case.edu

\author{
Shankar Subramaniam \\ Department of Bioengineering \\ University of California \\ San Diego \\ 9500 Gilman Dr. \\ La Jolla California 92093-0412 \\ USA \\ shankar@sdsc.edu
}

\author{
Ananth Grama \\ Department of Computer Science \\ Purdue University \\ 305 North University Street \\ West Lafayette 47907-2107 \\ Indiana \\ USA \\ ayg@cs.purdue.edu
}

ISBN 978-1-4614-0319-7

DOI 10.1007/978-1-4614-0320-3

Springer New York Dordrecht Heidelberg London

Library of Congress Control Number: 2011937231

(C) Springer Science+Business Media, LLC 2012

All rights reserved. This work may not be translated or copied in whole or in part without the written permission of the publisher (Springer Science+Business Media, LLC, 233 Spring Street, New York, NY 10013, USA), except for brief excerpts in connection with reviews or scholarly analysis. Use in connection with any form of information storage and retrieval, electronic adaptation, computer software, or by similar or dissimilar methodology now known or hereafter developed is forbidden.

The use in this publication of trade names, trademarks, service marks, and similar terms, even if they are not identified as such, is not to be taken as an expression of opinion as to whether or not they are subject to proprietary rights.

Printed on acid-free paper

Springer is part of Springer Science+Business Media (www.springer.com) 


\section{Preface}

The past decade has seen tremendous advances in our understanding of the basic mechanisms that underlie living organisms. These advances have been motivated by our ability to gather large amounts of data relating to the state of living systems (chemical compositions, dynamic fluxes, binding states, etc.), modeling in ways that lend themselves to powerful analyses techniques, and associated algorithms and software. In contrast to traditional approaches that take a deconstructive view of biological processes (scaling up from atomistic and molecular levels), systems biology studies the organization and emergent properties of interacting units (typically biomolecules). This poses profound challenges, as well as exciting opportunities for new discoveries. This edited volume focuses on the computational challenges associated with systems-level modeling of biological function and explores how functional relationships among biomolecules are manifested in biological networks.

Systems-level (network) models of biological systems typically rely on graphs with nodes corresponding to biological entities and edges corresponding to interrelationships among these entities. Constructing such models from data, reasoning from these models, characterizing their organization and function, and relating them to the genotype/phenotype, pose complex modeling and algorithmic challenges. While one may be tempted to view these as traditional graph algorithms, well studied in computer science literature, the unique characteristics of biological interaction data necessitates development of novel models and methods. These characteristics include: (1) incomplete and noisy datasets, (2) highly skewed distributions, (3) need for establishing statistical validity, (4) incorporating elements of space, time, and scale, and (5) relating across disparate abstractions.

While there is tremendous ongoing research and development activity in this area, there is an established core of results that provides the basis for future development. This book provides a comprehensive overview of the state-of-the-art, as it relates to modeling and analysis of living systems as interacting biological units. By the nature of the underlying processes, the book intersects broad subdisciplines within biology, statistics, and computer science. It addresses methods for data collection and curation, model inference, statistically grounded analyses, 
algorithms, software and frameworks, and validation. It also motivates a number of open problems, which provide excellent avenues for continuing efforts in the area.

The book is the result of significant efforts on part of the contributing authors. The editors would like to thank all of the authors for their outstanding contributions.

Cleveland, OH, USA

West Lafayette, IN, USA

Mehmet Koyutürk

La Jolla, CA, USA

Ananth Grama

Shankar Subramaniam 


\section{Contents}

1 Introduction to Network Biology .............................. 1

Mehmet Koyutürk, Shankar Subramaniam, and Ananth Grama

2 Topological Characteristics of Molecular Networks ................. 15

Tijana Milenković and Nataša Pržulj

3 Function Annotation in Gene Networks ........................ 49

Petko Bogdanov, Kathy Macropol, and Ambuj K. Singh

4 Proteome Network Emulating Models............................. 69

Phuong Dao, Fereydoun Hormozdiari, Iman Hajirasouliha,

Martin Ester, and S. Cenk Sahinalp

5 Biological Network Alignment ................................. 97

Shahin Mohammadi and Ananth Grama

6 Pattern Mining Across Many Massive Biological Networks ........... 137

Wenyuan Li, Haiyan Hu, Yu Huang, Haifeng Li, Michael

R. Mehan, Juan Nunez-Iglesias, Min Xu, Xifeng Yan, and Xianghong Jasmine Zhou

7 Molecular Networks and Complex Diseases

Mehmet Koyutürk, Sinan Erten, Salim A. Chowdhury,

Rod K. Nibbe, and Mark R. Chance

8 Moving Toward Genome-Scale Kinetic Models: The Mass

Action Stoichiometric Simulation Approach ...................... 201

Aarash Bordbar and Bernhard Ø. Palsson

Index

221 



\section{Contributors}

Petko Bogdanov Department of Computer Science, University of California, Santa Barbara, CA 93106, USA, petko@cs.ucsb.edu

Aarash Bordbar University of California, San Diego, CA 92093-5004, USA, aabordba@ucsd.edu

Mark R. Chance Case Western Reserve University, Cleveland, OH 44106-3808, USA,mrc16@case.edu

Salim A. Chowdhury Carnegie-Mellon University, Pittsburgh, PA 15213, USA, sachowdh@andrew.cmu.edu

Phuong Dao School of Computing Science, Simon Fraser University, 8888 University Drive, Burnaby, B.C., V5A 1S6 Canada, pdao@cs.sfu.ca

Sinan Erten Case Western Reserve University, Cleveland, OH 44106-3808, USA, mse10@case.edu

Martin Ester Simon Fraser University, Burnaby, B.C., V5A 1S6 Canada, ester@cs.sfu.ca

Ananth Grama Department of Computer Science, Purdue University, 305 N. University Street, West Lafayette, IN 47907-2107, USA,

ayg@purdue.edu

Iman Hajirasouliha Simon Fraser University, Burnaby, B.C., V5A 1S6 Canada, imanh@cs.sfu.ca

Fereydoun Hormozdiari Simon Fraser University, Burnaby, B.C., V5A 1S6 Canada, fhormozd@cs.sfu.ca

Haiyan Hu University of Central Florida, 4000 Central Florida Boulevard Orlando, FL 32816, USA, haihu@cs.ucf.edu

Yu Huang University of Southern California, Los Angeles, CA 90007-2439, USA, yuhuang@usc.edu 
Mehmet Koyutürk Department of Electrical Engineering and Computer Science, Case Western Reserve University, 10900 Euclid Ave., Cleveland, OH 44106, USA, mxk331@case.edu

Haifeng Li Motorola Labs, Los Angeles, CA 90013, USA, haifeng1@usc.edu

Wenyuan Li University of Southern California, Los Angeles, CA 90007-2439, USA,wel@usc.edu

Kathy Macropol University of California, Santa Barbara, Santa Barbara, CA 93106, USA, kpm@cs.ucsb.edu

Michael R. Mehan University of Southern California, Los Angeles, CA 900072439, USA, rielmeha@usc.edu

Tijana Milenković Department of Computer Science and Engineering, University of Notre Dame, Notre Dame, IN 46556, USA, tmilenko@nd.edu

Shahin Mohammadi Purdue University, Lafayette, IN 47906, USA, mohammas@purdue.edu

Rod K. Nibbe Case Western Reserve University, Cleveland, OH 44106-3808, USA,rkn6@case.edu

Juan Nunez-Iglesias University of Southern California, Los Angeles, CA 900072439, USA, nunezigl@usc.edu

Bernhard Ø. Palsson Department of Bioengineering, University of California San Diego, 9500 Gilman Dr., La Jolla, CA, USA, palsson@ucsd.edu

Nataša Pržulj Imperial College London, Exhibition Rd, London SW7 2AZ, UK, natasha@imperial.ac.uk

S. Cenk Sahinalp Simon Fraser University, Burnaby, B.C., V5A 1S6 Canada, cenk@cs.sfu.ca

Ambuj K. Singh University of California, Santa Barbara, CA 93106, USA, ambuj@cs.ucsb.edu

Shankar Subramaniam University of California, San Diego, CA 92093-5004, USA, shankar@sdsc.edu

Min Xu University of Southern California, Los Angeles, CA 90007-2439, USA, mxu@usc.edu

Xifeng Yan University of California, Santa Barbara, CA 93106, USA, xyan@cs.ucsb.edu

Xianghong Jasmine Zhou Program in Computational Biology, Department of Biological Sciences, University of Southern California, Los Angeles, CA 90089, USA, xjzhou@usc.edu 